\title{
Synthesis of DNA oligonucleotides containing 5-(mercaptomethyl)-2'-deoxyuridine moieties
}

\author{
Benjamin Bornemann and Andreas Marx* \\ Fachbereich Chemie, Universität Konstanz, Universitätsstrasse 10, 78457 Konstanz, Germany
}

\begin{abstract}
Recently thiolated oligonucleotides have attracted significant interest due to their ability to efficiently undergo stable bond formation with gold nanoparticles and surfaces to form DNA conjugates. In this respect we became interested in the synthesis of oligonucleotides that bear short thioalkyl functions located at the nucleobase. Here we present a strategy for the synthesis of DNA oligonucleotides that bear 5 (mercaptomethyl) $2^{\prime}$ deoxyuridine moieties. The building blocks were synthesized in a straight forward manner from thymidine. Only moderate changes of standard protocols for automated DNA synthesis are required for the generation of modified oligonucleotides containing the thiolated building blocks.
\end{abstract}

\section{Introduction}

Efficient chemical modification of DNA and RNA oligonucleotides is the basis for numerous subsequent studies like conjugation of reporter molecules, investigation of biological systems or immobilization on surfaces. ${ }^{1,2}$ Among the numerous functionalities conjugated to DNA, thiol moieties are of particular interest, for example, for conjugation of reporter groups, immobilization of DNA oligonucleotides on gold surfaces or nanoparticles. ${ }^{1-7}$ In most current studies thioalkyl functions are coupled to the $5^{\prime}$ - or $3^{\prime}$-end of the oligonucleotides, respectively, employing thioalkylated phosphoramidites or solid supports. ${ }^{8,9}$ Recently, oligonucleotides that were thioalkylated at the $3^{\prime}$-terminus were employed in studies dedicated to directly measure electrical transport through single DNA molecules. ${ }^{10,11}$ To fulfill these tasks, respective oligonucleotide duplexes were equipped with thioalkyl functions and employed to form double stranded DNA (dsDNA) bridges between two gold contacts. In this respect we became interested in the synthesis of oligonucleotides that bear short thioalkyl functions in close proximity to the $\pi$-system of the nucleobase to facilitate electrical transport through dsDNA. Thus, our first aim was the synthesis of DNA

Keywords: DNA; Thiol functions; Conjugation; Oligonucleotide; Nucleoside; Phosphoramidite; Gold.

* Corresponding author. Tel.: +49 0753188 5139; fax: +490 0753188

5140; e mail: Andreas.Marx@uni konstanz.de oligonucleotides that bear 5-(mercaptomethyl)- $2^{\prime}$-deoxyuridine moieties (Fig. 1). We intended to develop a strategy that allows positioning of the thiolated building blocks at the $3^{\prime}$ - or $5^{\prime}$-terminal nucleotide or at internal locations to ensure maximum flexibility.

Recently, a route for the synthesis of oligonucleotides containing 5-(mercaptomethyl)-2'-deoxyuridine moieties was developed. ${ }^{12}$ The reported approach harbors several disadvantages like the usage of organomercury species and comparatively expensive starting materials. Here we present an improved and concise strategy for the synthesis of oligonucleotides that bear 5-(mercaptomethyl)$2^{\prime}$-deoxyuridine moieties in principle at any desired position. The herein depicted approach relies on the selective activation of the 5-methyl group of thymidines through bromination and the subsequent nucleophilic replacement of bromide by thioacetate.
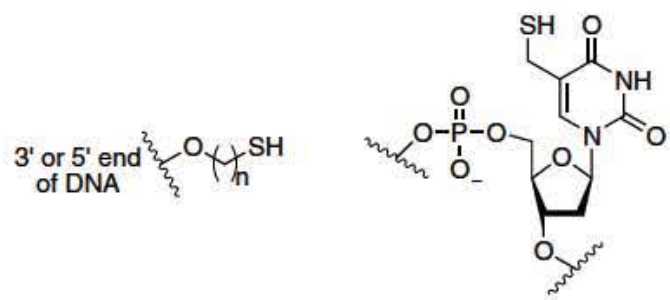

Figure 1. Thioalkyl functions conjugated to the $5^{\prime}$ or $3^{\prime}$ end of DNA (left), 5 (mercaptomethyl) $2^{\prime}$ deoxyuridine moieties incorporated into DNA oligonucleotides. 


\section{Results and discussion}

\subsection{Nucleoside synthesis}

Our synthesis of suitable building blocks for the synthesis of modified DNA oligonucleotides started with the $3^{\prime}, 5^{\prime}$-bis- $O$-(tert-butyl dimethylsilyl) protected thymidine derivative $1^{13}$ that was easily accessible from thymidine in high yields (Scheme 1).

Radical bromination in the benzylic position was achieved with $\mathrm{N}$-bromo succinimide (NBS) and 2,2'azo-bis-isobutyronitrile (AIBN) at elevated temperature. The resulting bromide 2 turned out to be rather labile and was thus used crude for further transformation after filtration of the reaction mixture. Substitution of bromide with potassium thioacetate afforded the protected 5-(mercaptomethyl)- $2^{\prime}$-deoxyuridine analog 3 . Selective $3^{\prime}, 5^{\prime}$-bis-O-desilylation was achieved smoothly to yield 4 under acidic conditions employing a 4:1 mixture of acetic acid $(75 \%)$ and THF. Noteworthy, attempts to synthesize $\mathbf{4}$ directly from thymidine without any protection of the nucleoside failed as well as cleavage of the silyl ethers of 3 employing tetrabutyl ammonium fluoride (TBAF).

\subsection{Synthesis of modified oligonucleotides}

Next modified nucleoside 4 was converted into building blocks suited for automated DNA synthesis (Scheme 2).

Thus, 4 was $5^{\prime}$-O-protected with $4,4^{\prime}$-dimethoxytrityl (DMT) employing standard conditions to yield 5 in very good yields. In order to obtain oligonucleotide strands 7 that bear modified thymidine residues at the 3 -terminal position, 5 was coupled to solid support to afford 6 . Employing solid support 6 oligonucleotides 7 were synthesized using standard $\beta$-cyanoethyl phosphoramidites and coupling conditions. However, the deprotection protocol had to be adjusted: After completion of the synthesis, solid supports were first treated with 1,8-diazabicyclo[5.4.0]undec-7-ene (DBU) and afterwards with concentrated ammonia in the presence of 1,4-dithiothreitol (DTT). In order to synthesize oligonucleotides that bear the 5-thiolated thymidine moiety at the 5 -terminal position or at an internal position, phosphoramidite 8 was synthesized by phosphitylation of $\mathbf{5}$ in good yields. Employing $\mathbf{8}$ oligonucleotides $\mathbf{9}$ were synthesized that contain the modified building block at the 5 '-terminal position or within the DNA strand. Table 1 lists the synthesized oligonucleotides and provides the analytical
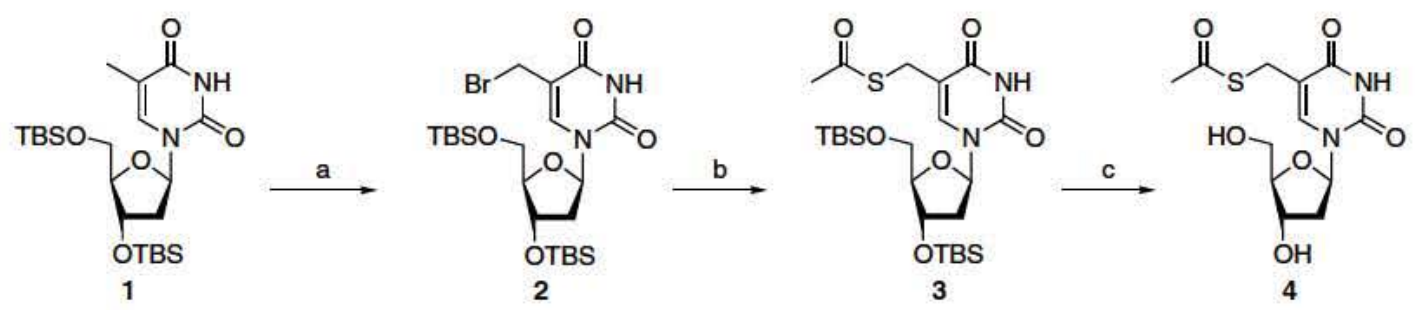

Scheme 1. Synthesis of 5 (mercaptomethyl) $2^{\prime}$ deoxyuridine. Reagents and conditions: (a) NBS, AIBN, $\mathrm{CCl}_{4}$, reflux, 2.5 h; (b) potassium thioacetate, DMF, $1.5 \mathrm{~h}$, (39\%, over two steps); (c) AcOH, THF, 24 h (73\%).

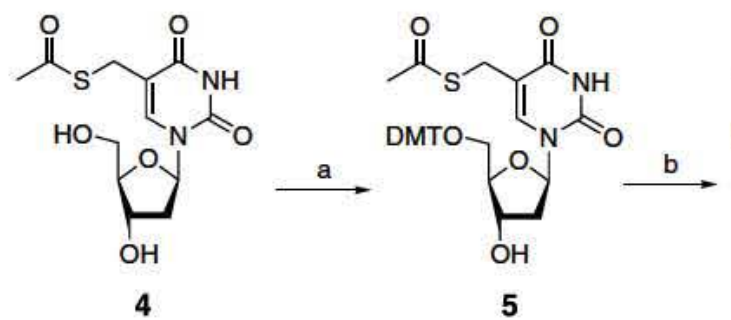<smiles>COCC1OC2CC1C(OC(=O)CCC(=O)O)C2n1cc(CSC(C)=O)c(=O)[nH]c1=O</smiles><smiles>CCCOP(=O)(O)OCC1C(O)CC2CC1O2</smiles>
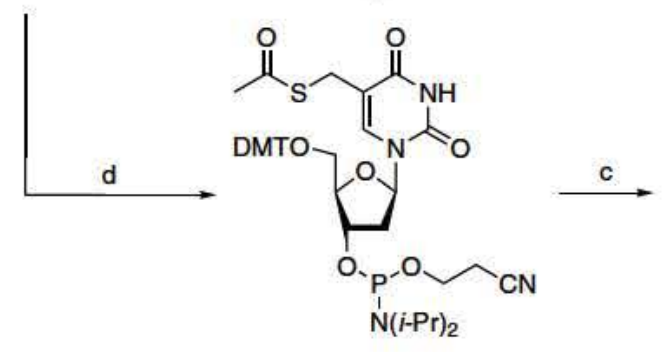

8

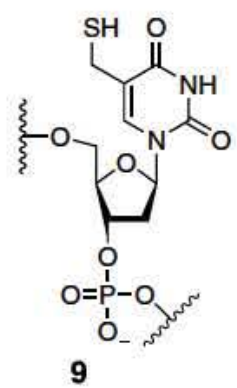

Scheme 2. Synthesis of DNA oligonucleotides containing 5 (mercaptomethyl) $2^{\prime}$ deoxyuridines. Reagents and conditions: (a) DMT Cl, DMAP, pyridine, $0^{\circ} \mathrm{C}, 24 \mathrm{~h},(90 \%)$; (b) EDC, DMAP, succinylated LCAA CPG, pyridine; then 4 nitrophenol; then piperidine, then acetic anhydride/ pyridine/THF (cap A) and 1 methylimidazole/THF (cap B); (c) i oligonucleotide synthesis, ii DBU, then $33 \% \mathrm{NH}_{4} \mathrm{OH}, 0.2 \mathrm{M}$ DTT; (d) $\left[\left({ }^{i} \mathrm{Pr}_{2} \mathrm{~N}\right)\left(\mathrm{NCCH}_{2} \mathrm{CH}_{2} \mathrm{O}\right) \mathrm{P}\right] \mathrm{Cl}, \mathrm{NEt}_{3}, \mathrm{CH}_{2} \mathrm{Cl}_{2}, \mathrm{rt}, 4 \mathrm{~h},(66 \%)$. 
Table 1. Synthesized DNA oligonucleotides

\begin{tabular}{llll}
\hline & Sequence & Calcd mass [M H] & \\
\hline S1 & 5' TTT TTT TTT TTT* 3' & 3619.4 & 3619.6 \\
S2 & 5' CGT TGG TCC TGA AGG AGG AT* 3' & 6244.1 & 6243.7 \\
S3 & 5' CGT TGG TCC T*GA AGG AGG AT 3' & 6244.1 & 6246.8 \\
S4 & 5' T*CG TTG GTC CTG AAG GAG GAT 3' & 6548.3 & 6551.1 \\
\hline
\end{tabular}

$\mathrm{T}^{*}, 5$ (mercaptomethyl) $2^{\prime}$ deoxyuridine.

data obtained by electrospray ionization mass spectrometer (ESI-MS).

\section{Conclusions}

Acetylated 5-(mercaptomethyl)-2'-deoxyuridine 4 was synthesized in good yields in a straightforward synthesis from thymidine. Compound $\mathbf{4}$ was subsequently converted into suitable building blocks for the automated synthesis of DNA oligonucleotides that contain the modified residues at desired positions. Through only moderate changes of standard protocols for automated DNA synthesis the generation of modified oligonucleotides containing the thiolated building blocks was feasible. Currently, we are investigating the properties of the synthesized oligonucleotides for immobilization on gold surfaces.

\section{Experimental}

\subsection{General}

All temperatures quoted are uncorrected. All reagents are commercially available and used without further purification. Solvents are purchased over molecular sieves (Fluka) and used directly without further purification unless otherwise noted. All reactions were conducted under rigorous exclusion of air and moisture. Elemental analysis was carried out by the microanalysis facility of the University of Konstanz. NMR spectra were recorded on Bruker AC 250 Cryospec $\left({ }^{1} \mathrm{H}\right.$ : $250 \mathrm{MHz})$, Jeol JNA-LA-400 $\left({ }^{1} \mathrm{H}: 400 \mathrm{MHz},{ }^{13} \mathrm{C}\right.$ : $100 \mathrm{MHz})$, and Bruker DRX $600\left({ }^{1} \mathrm{H}: 600 \mathrm{MHz},{ }^{13} \mathrm{C}\right.$ : $150 \mathrm{MHz}$ ). Chemical shifts are given in parts per million $(\delta)$ relative to the residual solvent signal. MALDI-TOF mass spectra were recorded on a Kompact MALDI II mass spectrometer (Kratos Analytical) in positive, linear mode with delayed extraction MALDI source and a nitrogen laser $(337 \mathrm{~nm})$. ESI-IT mass spectra were recorded on a Bruker Daltonics esquire 3000+ in positive or negative mode with a flow rate of $3 \mu \mathrm{l} / \mathrm{min}$. DNA oligonucleotides were synthesized on an Applied-Biosystems 392 DNA/RNA-synthesizer employing a standard phosphoramidite strategy. Flash chromatography: Merck silica gel G60 (230 400 mesh). Thinlayer chromatography: Merck precoated plates (silica gel $60 \mathrm{~F}_{254}$ ). Reversed-phase HPLC was performed on a prominence-line HPLC (Shimadzu) with a Nucleosil100-5-(250/4)-C18-column from Macherey-Nagel and a binary gradient system (TEAA-buffer $(0.1 \mathrm{M})$, acetonitrile).

4.1.1. 5-Bromomethyl-3', $5^{\prime}$-bis- $O$-(tert-butyldimethylsilyl)-2'-deoxyuridine (2). To a solution of $\mathbf{1}^{13}(1.00 \mathrm{~g}$,
$2.13 \mathrm{mmol})$ in dry $\mathrm{CCl}_{4}(20 \mathrm{ml})$ under argon atmosphere NBS (567 mg, $3.18 \mathrm{mmol}$ ) and AIBN (35 mg, $0.21 \mathrm{mmol}$ ) were added and heated at reflux for $2.5 \mathrm{~h}$. The brownish reaction mixture was then filtered through a sintered glass frit (D4) to remove the succinimide and evaporated to dryness. Compound $\mathbf{2}$ was used immediately without further purification.

\subsection{2. $S$-Acetyl-3', $5^{\prime}$-bis- $O$-(tert-butyldimethylsilyl)-5-} (mercaptomethyl)-2'-deoxyuridine (3). To a solution of crude $\mathbf{2}$ in dry DMF $(20 \mathrm{ml})$ under argon atmosphere potassium thioacetate $(1.00 \mathrm{~g}, 8.75 \mathrm{mmol})$ was added and heated to $75^{\circ} \mathrm{C}$ for $1.5 \mathrm{~h}$. The reaction mixture was diluted with $\mathrm{CH}_{2} \mathrm{Cl}_{2}(100 \mathrm{ml})$ and washed with water $(3 \times 50 \mathrm{ml})$. The organic phase was dried with magnesium sulfate and evaporated to dryness. The product 3 was purified by column chromatography (petrol ether ethyl acetate, $3: 1)$ to a yield of $444 \mathrm{mg}(39 \%$, over two steps). ${ }^{1} \mathrm{H}$ NMR $\left(250 \mathrm{MHz}, \mathrm{CDCl}_{3}\right): \delta 0.08(\mathrm{~s}, 6 \mathrm{H}$, $\left.\mathrm{Si}\left(\mathrm{CH}_{3}\right)_{2}\right), \quad 0.12 \quad\left(\mathrm{~s}, \quad 6 \mathrm{H}, \quad \mathrm{Si}\left(\mathrm{CH}_{3}\right)_{2}\right), \quad 0.89 \quad(\mathrm{~s}, \quad 9 \mathrm{H}$, $\left.\mathrm{SiC}\left(\mathrm{CH}_{3}\right)_{3}\right), 0.92\left(\mathrm{~s}, 9 \mathrm{H}, \mathrm{SiC}\left(\mathrm{CH}_{3}\right)_{3}\right), 1.922 .02(\mathrm{~m}, 1 \mathrm{H}$, $\left.\mathrm{H} 2^{\prime} \mathrm{A}\right), 2.222 .23\left(\mathrm{~m}, 1 \mathrm{H}, \mathrm{H} 2^{\prime} \mathrm{B}\right), 2.30\left(\mathrm{~s}, 3 \mathrm{H},(\mathrm{CO}) \mathrm{CH}_{3}\right)$, $3.75\left(\mathrm{~s}, 2 \mathrm{H}, \mathrm{CH}_{2} 5\right), 3.793 .84\left(\mathrm{~m}, 2 \mathrm{H}, \mathrm{H}^{\prime} \mathrm{A}^{\prime}\right.$ and $\left.\mathrm{H} 5^{\prime} \mathrm{B}\right)$, $3.923 .98\left(\mathrm{~m}, 1 \mathrm{H}, \mathrm{H} 4^{\prime}\right), 4.414 .46\left(\mathrm{~m}, 1 \mathrm{H}, \mathrm{H} 3^{\prime}\right), 6.28$ $\left(\mathrm{dd}, 1 \mathrm{H}, J=6.4 \mathrm{~Hz}\right.$ and $\left.6.4 \mathrm{~Hz}, \mathrm{H}^{\prime}{ }^{\prime}\right), 7,75$ (s, 1H, H6), 9.70 (br s, 1H, NH); MS (MALDI-TOF, DHB): Calcd for $\mathrm{C}_{24} \mathrm{H}_{44} \mathrm{~N}_{2} \mathrm{O}_{6} \mathrm{SSi}_{2}[\mathrm{M}+\mathrm{Na}]^{+}$: 567.24, $\mathrm{C}_{24} \mathrm{H}_{44} \mathrm{~N}_{2} \mathrm{O}_{6} \mathrm{SSi}_{2}$ $[\mathrm{M}+\mathrm{K}]^{+}:$583.22. Found: 567.1, 583.1.

4.1.3. S-Acetyl-5-(mercaptomethyl)-2'-deoxyuridine (4). Compound 3 (440 mg, $0.81 \mathrm{mmol}$ ) was deprotected in a mixture $(20 \mathrm{ml})$ of $75 \%$ acetic acid and THF (4:1). The reaction was monitored by TLC $\left(\mathrm{CH}_{2} \mathrm{Cl}_{2}\right.$ methanol, 95:5). After complete conversion $(24 \mathrm{~h})$, the reaction mixture was evaporated to dryness and purified by column chromatography $\left(\mathrm{CH}_{2} \mathrm{Cl}_{2}\right.$ methanol, 95:5) to yield $187 \mathrm{mg}(73 \%)$ of 4. ${ }^{1} \mathrm{H}$ NMR $\left(250 \mathrm{MHz}, \mathrm{MeOH}-d_{4}\right) \delta$ $2.072 .23\left(\mathrm{~m}, 2 \mathrm{H}, \mathrm{H} 2^{\prime} \mathrm{A}\right.$ and $\left.\mathrm{H} 2^{\prime} \mathrm{B}\right), 2.35$ (s, 3H, $\left.(\mathrm{CO}) \mathrm{CH}_{3}\right), 3.713 .86\left(\mathrm{~m}, 4 \mathrm{H}, \mathrm{H}^{\prime} \mathrm{A}, \mathrm{H} 5^{\prime} \mathrm{B}\right.$ and $\left.\mathrm{SCH}_{2}\right)$ $3.93\left(\mathrm{dt}, 1 \mathrm{H}, J=3.7 \mathrm{~Hz}\right.$ and $\left.4.3 \mathrm{~Hz}, \mathrm{H}^{\prime}\right), 4.41(\mathrm{dt}$, $1 \mathrm{H}, \quad J=2.7 \mathrm{~Hz}$ and $\left.4.3 \mathrm{~Hz}, \mathrm{H} 3^{\prime}\right), 6.22(\mathrm{dd}, 1 \mathrm{H}$, $J=6.7 \mathrm{~Hz}$ and $\left.6.7 \mathrm{~Hz}, \mathrm{H}^{\prime}{ }^{\prime}\right), 8.00(\mathrm{~s}, 1 \mathrm{H}, \mathrm{H} 6)$; Calcd for $\mathrm{C}_{12} \mathrm{H}_{16} \mathrm{~N}_{2} \mathrm{O}_{6} \mathrm{~S}$ : C, 45.56; H, 5.10; N, 8.86. Found: C, 45.33; H, 5.19; N, 8.87; MS (ESI-MS, MeOH): Calcd for $\mathrm{C}_{12} \mathrm{H}_{16} \mathrm{~N}_{2} \mathrm{O}_{6} \mathrm{~S} \quad[\mathrm{M}+\mathrm{H}]^{+}: \quad 316.07, \quad \mathrm{C}_{12} \mathrm{H}_{16} \mathrm{~N}_{2} \mathrm{O}_{6} \mathrm{~S}$ $[\mathrm{M}+\mathrm{Na}]^{+}:$338.07. Found: 317.1, 338.1.

\subsection{4. $S$-Acetyl-5'-O-(dimethoxytrityl)-5-(mercaptometh-} yl)-2'-deoxyuridine (5). A solution of 4 (180 mg, $0.57 \mathrm{mmol})$ in dry pyridine $(10 \mathrm{ml})$ under argon atmosphere was cooled to $0^{\circ} \mathrm{C}$, dimethoxytritylchloride (212 $\mathrm{mg}, 0.63 \mathrm{mmol}$ ) and DMAP (7 mg, $0.06 \mathrm{mmol}$ ) were added. The reaction was kept at $0{ }^{\circ} \mathrm{C}$ for $24 \mathrm{~h}$ and then quenched with $1 \mathrm{ml}$ methanol. The solution 
was evaporated to dryness, dissolved in $\mathrm{CH}_{2} \mathrm{Cl}_{2}(10 \mathrm{ml})$, and washed with saturated $\mathrm{NaHCO}_{3}$ solution. The organic phase was dried with magnesium sulfate, evaporated to dryness, and purified by column chromatography $\left(1 \% \mathrm{Et}_{3} \mathrm{~N}\right.$ in ethyl acetate) to yield $317 \mathrm{mg}(90 \%)$ of $5 .{ }^{1} \mathrm{H}$ NMR (400 MHz, acetone- $\left.d_{6}\right): \delta 2.062 .09(\mathrm{~m}$, $2 \mathrm{H}, \mathrm{H} 2^{\prime} \mathrm{A}$ and $\left.\mathrm{H} 2^{\prime} \mathrm{B}\right), 2.20\left(\mathrm{~s}, 3 \mathrm{H},(\mathrm{CO}) \mathrm{CH}_{3}\right), 3.29$ $3.55\left(\mathrm{~m}, 4 \mathrm{H}, \mathrm{H}^{\prime} \mathrm{A}, \mathrm{H} 5^{\prime} \mathrm{B}\right.$ and $\left.\mathrm{SCH}_{2}\right), 3.79(\mathrm{~s}, 6 \mathrm{H}$, $\left.\mathrm{OCH}_{3}\right), 4.06\left(\mathrm{dt}, 1 \mathrm{H}, J=4.0 \mathrm{~Hz}\right.$ and $\left.4.4 \mathrm{~Hz}, \mathrm{H}^{\prime}\right), 4.51$ $\left(\mathrm{dt}, 1 \mathrm{H}, J=4.0 \mathrm{~Hz}\right.$ and $\left.6.0 \mathrm{~Hz}, \mathrm{H}^{\prime}\right), 6.29(\mathrm{dd}, 1 \mathrm{H}$, $J=6.6 \mathrm{~Hz}$ and $\left.6.6 \mathrm{~Hz}, \mathrm{H}^{\prime}\right), 6.89(\mathrm{~d}, 4 \mathrm{H}, J=9.0 \mathrm{~Hz}$, Ar), $7.23(\mathrm{t}, 1 \mathrm{H}, J=7.3 \mathrm{~Hz}, \mathrm{Ar}), 7.32(\mathrm{dd}, 2 \mathrm{H}$, $J=7.3 \mathrm{~Hz}$ and $7.5 \mathrm{~Hz}, \mathrm{Ar}), 7.38(\mathrm{~d}, 2 \mathrm{H}, J=9.0 \mathrm{~Hz}$, Ar), $7.39(\mathrm{~d}, 2 \mathrm{H}, J=9.0 \mathrm{~Hz}, \mathrm{Ar}), 7.51(\mathrm{~d}, 2 \mathrm{H}$, $J=8.5 \mathrm{~Hz}, \mathrm{Ar}), 7.77$ (s, 1H, H6), 10.25 (br s, 1H, $\mathrm{NH}) ;{ }^{13} \mathrm{C}$ NMR $\left(100 \mathrm{MHz}\right.$, acetone- $\left.d_{6}\right): \delta 42.0\left(\mathrm{C} 2^{\prime}\right)$, $56.5\left(\mathrm{OCH}_{3}\right), 65.8\left(\mathrm{C}^{\prime}\right), 73.0\left(\mathrm{C}^{\prime}\right), 86.6\left(\mathrm{Cl}^{\prime}\right), 88.0$ (C4'), $88.3\left(\mathrm{CAr}_{3}\right), 111.9$ (C5), 115.0 (Ar), 127.2 (Ar), 128.7 (Ar), 129.8 (Ar), 130.1 (Ar), 130.9 (Ar), 132,1 (Ar), 132.1 (Ar), 137.8 (C6), 147.1 (C2) 160.8 (C4), 196.8 (CO); MS (ESI-MS, acetone): Calcd for $\mathrm{C}_{33} \mathrm{H}_{34} \mathrm{~N}_{2} \mathrm{O}_{8} \mathrm{~S} \quad[\mathrm{M}+\mathrm{H}]^{+}: \quad 619.20, \quad \mathrm{C}_{33} \mathrm{H}_{34} \mathrm{~N}_{2} \mathrm{O}_{8} \mathrm{~S}$ $[\mathrm{M}+\mathrm{Na}]^{+}: \quad 641.20, \quad \mathrm{C}_{33} \mathrm{H}_{34} \mathrm{~N}_{2} \mathrm{O}_{8} \mathrm{~S} \quad[\mathrm{M}+\mathrm{K}]^{+}: \quad 657.18$. Found: 619.1, 641.1, 657.1.

4.1.5. $S$-Acetyl-4'-O-(2-cyanoethoxy)(diisopropylamino)phosphino-5'-O-(dimethoxytrityl)-5-(mercaptomethyl)-2'-deoxyuridine (6). To a solution of 5 (100 mg, $0.162 \mathrm{mmol})$ in dry $\mathrm{CH}_{2} \mathrm{Cl}_{2}(5 \mathrm{ml})$ under argon atmosphere triethylamine $(82 \mathrm{mg}, 0.81 \mathrm{mmol})$ and 2-cyanoethyl- $N, N$-diisopropylchlorophosphoramidite $\quad(77 \mathrm{mg}$, $0.32 \mathrm{mmol})$ were added. The reaction was monitored by TLC (petrol ether ethyl acetate, 1:2). After complete conversion $(4 \mathrm{~h})$, the reaction mixture was evaporated to dryness and purified by column chromatography $(1 \%$ $\mathrm{Et}_{3} \mathrm{~N}$ in petrol ether ethyl acetate, $\left.1: 2\right)$ to yield $88 \mathrm{mg}$ $(66 \%)$ of $6 .{ }^{1} \mathrm{H}$ NMR $\left(600 \mathrm{MHz}\right.$, acetone- $\left.d_{6}\right): \delta 1.18$ $\left(\mathrm{d}, 6 \mathrm{H}, J=7.5 \mathrm{~Hz}, \quad \mathrm{NCH}\left(\mathrm{CH}_{3}\right)\right), 1.20(\mathrm{~d}, 6 \mathrm{H}$, $\left.J=7.5 \mathrm{~Hz}, \mathrm{NCH}\left(\mathrm{CH}_{3}\right)\right), 2.20\left(\mathrm{~s}, 3 \mathrm{H},(\mathrm{CO}) \mathrm{CH}_{3}\right), 2.36$ $2.48\left(\mathrm{~m}, 2 \mathrm{H}, \mathrm{H} 2^{\prime} \mathrm{A}\right.$ and $\left.\mathrm{H} 2^{\prime} \mathrm{B}\right), 2.64(\mathrm{t}, 2 \mathrm{H}, J=5.9 \mathrm{~Hz}$, $\left.\mathrm{CH}_{2} \mathrm{CN}\right), 3.443 .48\left(\mathrm{~m}, 2 \mathrm{H}, \mathrm{H}^{\prime} \mathrm{A}\right.$ and $\left.\mathrm{H}^{\prime} \mathrm{B}\right), 3.62$ $3.68\left(\mathrm{~m}, 2 \mathrm{H}, \mathrm{CH}_{2} 5\right), 3.703 .76(\mathrm{~m}, 2 \mathrm{H}, \mathrm{N}(\mathrm{CH})), 3.80$ $\left(\mathrm{s}, 6 \mathrm{H}, \mathrm{OCH}_{3}\right), 4.174 .22\left(\mathrm{~m}, 1 \mathrm{H}, \mathrm{H}^{\prime}\right), 4.664 .73(\mathrm{~m}$, $\left.1 \mathrm{H}, \mathrm{H}^{\prime}\right), 6.29\left(\mathrm{dd}, 1 \mathrm{H}, J=6.6 \mathrm{~Hz}\right.$ and $\left.6.6 \mathrm{~Hz}, \mathrm{H}^{\prime}\right)$, $6.91(\mathrm{~d}, 4 \mathrm{H}, J=9.0 \mathrm{~Hz}, \operatorname{Ar}), 7.25(\mathrm{t}, 1 \mathrm{H}, J=7.3 \mathrm{~Hz}$, Ar), 7.34 (dd, $2 \mathrm{H}, J=7.7 \mathrm{~Hz}$ and $7.7 \mathrm{~Hz}, \mathrm{Ar}), 7.41$ (d, $4 \mathrm{H}, J=8.9 \mathrm{~Hz}, \mathrm{Ar}), 7.53(\mathrm{~d}, 2 \mathrm{H}, J=7.3 \mathrm{~Hz}, \mathrm{Ar})$, 7.80 (s, 1H, H6), 10.16 (br s, $1 \mathrm{H}, \mathrm{NH}) ;{ }^{13} \mathrm{C} \mathrm{NMR}$ $\left(150 \mathrm{MHz}, \quad\right.$ acetone- $\left.d_{6}\right): \quad \delta \quad 20.6 \quad\left(\mathrm{CH}_{2} \mathrm{CN}\right), \quad 24.4$ $\left(\mathrm{CH}\left(\mathrm{CH}_{3}\right)_{2}\right), 26.5\left(5-\mathrm{CH}_{2}\right), 30.1\left((\mathrm{CO}) \mathrm{CH}_{3}\right), 40.0\left(\mathrm{C}^{\prime}\right)$, $43.9(\mathrm{NCH}), 44.0(\mathrm{NCH}), 55.0\left(\mathrm{OCH}_{3}\right), 64.1\left(\mathrm{C5}^{\prime}\right)$, $74.0\left(\mathrm{C}^{\prime}\right), 85.7\left(\mathrm{Cl}^{\prime}\right), 86.1\left(\mathrm{C}^{\prime}\right), 87.3\left(\mathrm{CAr}_{3}\right), 110.9$ (C5), 114.0 (Ar), $118.8(\mathrm{CN}), 127.6$ (Ar), 128.7 (Ar), 129.1 (Ar), 131.1 (Ar), 136.6 (C6), 138.9 (Ar), 145.9 (Ar), 150.8 (C2), 159.7 (Ar), 162.9 (C4), 195.5 (CO); MS (ESI-MS, acetone): Calcd for $\mathrm{C}_{42} \mathrm{H}_{51} \mathrm{~N}_{4} \mathrm{O}_{9} \mathrm{PS}$ $[\mathrm{M}+\mathrm{Na}]^{+}: \quad 841.31, \mathrm{C}_{42} \mathrm{H}_{51} \mathrm{~N}_{4} \mathrm{O}_{9} \mathrm{PS} \quad[\mathrm{M}+\mathrm{K}]^{+}: \quad 857.28$. Found: $842.0,857.9$.

\subsection{Synthesis of modified oligonucleotides}

For the synthesis of oligonucleotides $\mathbf{S 1}$ and $\mathbf{S 2}$ solidphase support $\mathbf{6}$ was used, in all other cases the modified phosphoramidite 8 ( $0.1 \mathrm{M}$ in acetonitrile). The synthesis of oligonucleotides was carried out on a DNA-synthesizer on $0.2 \mu \mathrm{mol}$ scale applying commercially available 2-cyanoethylphosphoramidites. A standard method for 2-cyanoethylphosphoramidites was used, with the exception that the coupling time from the modified nucleotides was extended to $10 \mathrm{~min}$. Yields for modified oligonucleotides are comparable to those obtained for unmodified oligonucleotides. The synthesized oligonucleotide sequences are listed in Table 1. Following deprotection strategy was used: The solid-phase support was treated with $1 \mathrm{ml}$ of $10 \%$ DBU in acetonitrile for $30 \mathrm{~min}$ followed by washing with $5 \mathrm{ml}$ acetonitrile. The deprotection with ammonium hydroxide $(33 \%)$ occurred in the presence of excess DTT $(0.2 \mathrm{M})$ at $56^{\circ} \mathrm{C}$ for $16 \mathrm{~h}$. This deprotection procedure leads to the desired product and a minor product with a mass $120 \mathrm{Da}$ higher, presumably the DTT-adduct, which can be easily separated by HPLC. The oligonucleotides were purified by RPHPLC with a binary gradient system (A: 0.1 M TEAA-buffer and B: acetonitrile) and a gradient of 5 $20 \%$ B over $25 \mathrm{~min}$. Integrities of all modified oligonucleotides were confirmed by ESI MS.

\section{Acknowledgments}

We gratefully acknowledge financial support by the DFG within the framework of the SFB 513. The assistance of Dr. Karl-Heinz Jung in manuscript preparation is kindly acknowledged.

\section{References and notes}

1. Bioconjugation Protocols (Methods in Molecular Biology Series Vol. 283), Niemeyer, C. M., Ed.; Humana Press: Totowa, 2004.

2. DNA and Aspects of Molecular Biology (Comprehensive Natural Products Chemistry Vol. 7); Kool, E. T., Ed.; Pergamon Press: Oxford, 2002.

3. Beaucage, S. L.. In DNA and Aspects of Molecular Biology (Comprehensive Natural Products Chemistry); Kool, E. T., Ed.; Pergamon Press: Oxford, 2002; Vol. 7, pp 153249.

4. Bain, C. D.; Whitesides, G. M. Angew. Chem., Int. Ed. Engl. 1989, 28, 506.

5. Mirkin, C. A.; Letsinger, R. L.; Mucic, R. C.; Storhoff, J. J. Nature 1996, 382, 607.

6. Alivisatos, A. P.; Johnsson, K. P.; Peng, X.; Wilson, T. E.; Loweth, C. J.; Bruchez, M. P., Jr.; Schultz, P. G. Nature 1996, 382, 609 .

7. Pale Grosdemange, C.; Simon, E. S.; Prime, K. L.; Whitesides, G. M. J. Am. Chem. Soc. 1991, 113, 12.

8. Connolly, B. A.; Rider, P. Nucl. Acids Res. 1985, 13, 4485.

9. Sinha, N. D.; Cook, R. M. Nucl. Acids Res. 1988, 16, 2659.

10. Cohen, H.; Nogues, C.; Naaman, R.; Porath, D. Proc. Natl. Acad. Sci. U.S.A. 2005, 102, 11589.

11. Nogues, C.; Cohen, S. R.; Daube, S. S.; Naaman, R. Phys. Chem. Chem. Phys. 2004, 6, 4459.

12. Goodwin, J. T.; Glick, G. D. Tetrahedron Lett. 1993, 34, 5549.

13. Negron, G.; Calderon, G.; Vazquez, F.; Lomas, L.; Cardenas, J.; Marquez, C.; Gavino, R. Synth. Commun. 2002, 32, 1977. 